\title{
Método para aplicação de escâner a laser terrestre ao estudo da estabilidade de taludes em rocha
}

\author{
André Nagalli, Alberto Pio Fiori ${ }^{2}$ \& Bruno Nagalli ${ }^{3}$
}

\begin{abstract}
Resumo A utilização de técnicas de imageamento digital em geologia é uma tendência atual. O presente estudo buscou definir um método para aplicação de escâner a laser terrestre no estudo da estabilidade de taludes em rocha. Trata-se de uma tecnologia de uso recente cujos resultados na área geotécnica são ainda iniciais. A área escolhida para aplicação do método é a mina Saivá, situada no município de Rio Branco do Sul, Estado do Paraná, uma jazida de calcário explorada para a fabricação de cimento. O método de análise envolveu levantamentos de campo expeditos, identificação e medição de estruturas geológicas em imagem digital tridimensional, elaboração de diagramas estruturais e análise da estabilidade dos taludes. Os resultados obtidos revelam que a ferramenta mostra-se bastante vantajosa e precisa na medição de atitudes de planos de ruptura. A amplitude de variação das medidas de atitudes tomadas digitalmente é compatível com as observadas em campo e a possibilidade de se trabalhar simultaneamente grande quantidade de dados mostra-se um diferencial. Outra grande vantagem da técnica é a possibilidade de se obter parâmetros estruturais de áreas que em campo não se tem acesso direto, por questão de segurança ou fixação, por exemplo.
\end{abstract}

Palavras-chave: Estabilidade de taludes, Maciço Rochoso, Escâner a laser.

\begin{abstract}
Slope stability analysis using a terrester laser scanning method. The use of digital imaging techniques in geology is a current trend. This study intends to point a method to investigate the slope stability in rock masses trough images obtained by a terrester laser scanning system. Its application to the geotechnical uses is relatively incipient. The first stage of the study verified the applicability of the technique and its limitations. The object of the study was the Saivá mine, located in the city of Rio Branco do Sul, Paraná State, a pertaining calcareous rock deposit for cement manufacture. The analysis method involved field surveys, identification and measurement of geologic structures in three-dimensional digital image, elaboration of structural diagrams, classification of the rock mass, and analysis of the slope stability properly said. The results show that the tool may achieve accurate measurements of rupture plans, similar to the field measurements. Another advantage is that the method permits to work with large number of information simultaneously. One of the great advantages of the method is the possibility of getting structural parameters of areas that in field do not have direct access, for security or setting problems, for example.
\end{abstract}

Keywords: Slope stability, Rock masses, Terrester laser scanning.

INTRODUÇÃO O estudo da estabilidade de taludes possibilita a otimização de lavras em áreas de mineração, a segurança de cortes de estradas, túneis e encostas naturais, entre outros fatores. A instabilização de taludes em rocha se dá, em geral, pela conjugação de diversos fatores, que corroboram para o colapso da estrutura e envolvem aspectos extrínsecos (terremotos, sobrecargas, gradientes térmicos, etc.) e intrínsecos (características geométricas e constituintes dos maciços rochosos, distribuição espacial das descontinuidades, percolação de fluidos, etc.).

$\mathrm{O}$ avanço da ciência tem permitido, em especial com o uso de computadores, a aquisição e o tratamento simultâneo de uma quantidade cada vez maior de dados. Este fato possibilita análises estatisticamente mais acuradas e, conseqüentemente, resultados mais precisos.

O objetivo geral do presente trabalho é definir um método de investigação e predição da estabilidade de taludes em rocha a partir de imagens tridimensionais obtidas através de um escâner a laser terrestre. Embora tal equipamento tenha já sido utilizado na área da geotecnia para fins de monitoramento de encostas, o estudo paramétrico/geométrico das estruturas geológicas para fins geotécnicos, com esta tecnologia, não foi evidenciado na bibliografia consultada. A própria utilização deste equipamento é bastante recente e inovadora na área da geologia (Mezzomo 2007, Dalmolin \& Santos 2004; Nagalli et al. 2008).

Acrescenta-se a este fato a abordagem diferenciada que a técnica requer, pela interpretação e reconhecimento de uma determinada porção da nuvem de pontos como ente geológico-estrutural, inferindo-se direções preferenciais de descontinuidades, e levando-

1 - Programa de Pós-Graduação, Departamento de Geologia, Universidade Federal do Paraná, Curitiba (PR), Brasil.

E-mail: andrenagalli@yahoo.com.br

2 - Departamento de Geologia, Universidade Federal do Paraná, Curitiba (PR), Brasil. E-mail: fiori@ufpr.br

3 - Graduando do Curso de Geologia, Universidade Federal do Paraná, Curitiba (PR), Brasil. E-mail: brunonagalli@hotmail.com 
se em conta suas dimensões, freqüência, persistência, penetratividade, etc. Todos estes fatores podem influenciar diretamente o cálculo da estabilidade dos taludes.

REVISÃO BIBLIOGRÁFICA Avanços consideráveis foram conseguidos no estudo da susceptibilidade a fraturamento e sua modelagem, especialmente no que diz respeito ao campo de escorregamento no universo tridimensional e a seu monitoramento. Grande parte destes estudos emprega mapas topográficos digitais e modelos de elevação digital (DEM) derivados de sensores aéreos, ou seja, fotografias aéreas e equipamentos aerotransportados como o LIDAR (Light detection and ranging), por exemplo (Abellán et al. 2006, Pesci et al. 2007).

O inconveniente no emprego desta tecnologia decorre do fato destes sensores alcançarem densidades máximas de informação quando sua incidência é perpendicular à topografia, tipicamente superfícies subhorizontais. Por outro lado, as instabilidades devido ao fraturamento de rochas e seus deslocamentos usualmente ocorrem segundo planos verticais ou sub-verticais, justamente onde os resultados obtidos por meio de sensores terrestres são melhores (Armesto et al. 2009, Abellán et al. 2006).

O escâner a laser terrestre (TLS) é um equipamento capaz de capturar imagens e as coordenadas tridimensionais dos pontos que compõem a superfície de objetos, em um sistema de referência, com alta velocidade e acurácia. Staiger (2003) avaliou o desempenho de diferentes TLS disponíveis atualmente no mercado (dos fabricantes: Cyra, Mensi, Optech, Riegl e Z \& F), e concluiu que a performance dos equipamentos analisados é marcante, destacando-se uma aquisição de dados fácil e rápida, e boas precisões (em conformidade com o que estabelecem os fabricantes). A etapa de tratamento de dados foi considerada como limitante, pois consome grande parte do tempo do processo, requerendo maior automatização e agilidade.

Comparativamente a outras técnicas de medição terrestres como a estação total, o TLS apresenta como vantagem a rápida e automática obtenção, com acurácia (Pesci et al. 2007), de grande quantidade de dados, enquanto uma estação total, por exemplo, é dependente da habilidade do operador para a obtenção de uma quantidade de pontos que bem possam representar um afloramento, e este processo revela-se sobremaneira mais lento (Armesto et al. 2009). Outro ponto a favor do TLS é o fato deste permitir a aquisição de dados nas porções mais elevadas de um afloramento, possibilitando uma caracterização mais acurada, como por exemplo, a inclusão de melhores dados de persistência, orientação e curvatura de descontinuidades, o que possibilita uma base de dados para análise mais confiável (Sturzenegger \& Stead 2009).

A questão do tratamento dos dados obtidos junto ao escâner é o grande desafio atual, em função do alto potencial de aplicação da ferramenta sob diferentes abordagens. Os usos científicos atuais desta tecnologia abrangem suas mais diversas possibilidades, empregando-se o imageamento no estudo de esculturas e seu estado de preservação (Beraldin et al. 2000, Entwistle et al. 2009), análise de estruturas civis (Schueremans $\&$ Genechten 2009, Yoon 2009) e de infra-estrutura urbana (Barnea \& Filins 2008, Barber et al. 2008), análise, reconstrução e modelagem digital de arquitetura de árvores para fins de avaliação de madeira e desenvolvimento de folhas (Cote et al. 2009), preservação dos patrimônios artísticos, arqueológicos e históricos (Entwistle et al. 2009, Lerma et al. 2009), ferramenta de visão de robôs (Li et al. 2007), aplicações médicas (Meunier et al. 2000), usos industriais (Dalmolin \& Dos Santos 2004), entre outras.

No âmbito da geologia, o TLS tem seu uso ainda incipiente e sua utilização mostra-se restrita, com aplicações na área de fotogrametria (Mezzomo 2007, Pesci et al. 2007), no reconhecimento de feições estruturais e estratigráficas pela comparação com outras tecnologias de aerolevantamentos, por exemplo, no estudo da rugosidade de superfície em multiescalas (Fardin et al. 2004, Fardin et al. 2001) e no reconhecimento / diferenciação de litotipos (mármores e argilas) utilizando-se dados de intensidade de um escâner (Franceschi et al. 2009).

No âmbito da geotecnia e da geomorfologia, as principais aplicações do escâner a laser são o monitoramento de escorregamentos de taludes em solo e análise reversa (Dunning et al. 2009, Paronuzzi \& Serafini 2009), a obtenção de modelos digitais de terrenos (Pesci et al. 2007) e, subordinadamente acontecem estudos para o reconhecimento de formas (Armesto et al. 2009) e feições estruturais (Lato et al. 2009) para fins geomecânicos.

Armesto et al. (2009) empregaram o TLS para o reconhecimento de contornos de corpos graníticos (matacões) e suas propriedades geométricas e físicas (área de superfície, volume, centro de gravidade, etc.), visando a análise da estabilidade destes blocos a tombamentos e deslizamentos, considerando aspectos mecânicos e ambientais (intemperismo) para o cálculo do coeficiente de segurança do sistema.

Basicamente, o método de escanerização a laser consiste na emanação de feixes de luz (pulsos de laser) com comprimento de onda no campo ótico, ou próximo do domínio infravermelho, os quais atingem diretamente o objeto (Dalmolin \& Dos Santos 2004). A distância entre o ponto de emissão do laser (equipamento) e o objeto é calculada através da medida do tempo de retorno do sinal emitido, inferindo-se suas distâncias e ângulos. É então estabelecido um sistema de coordenadas cilíndricas tendo-se o equipamento no centro (origem) deste, permitindo assim calcular as respectivas coordenadas $\mathrm{X}, \mathrm{Y}$ e $\mathrm{Z}$ da cada ponto da nuvem de pontos obtida (Armesto et al. 2009).

A predição da estrutura de um maciço rochoso é uma importante ferramenta da engenharia de minas uma vez que o comportamento dessa massa de rocha pode ser controlado pela presença de descontinuidades (Turanboy et al. 2008). Segundo Fiori \& Carmignani (2009), são condições a serem observadas no processo de deslizamento planar: i) em condições drenadas, o ângulo de deslizamento deve ser maior que o ângulo de atrito daquele plano; ii) a direção do deslizamento 
deverá afastar-se de, no máximo, 20 graus em relação ao rumo de mergulho da face da vertente; iii) o plano de deslizamento deverá aflorar na face da vertente.

A análise de tombamento de blocos deve observar: i) deverá haver duas famílias de descontinuidades que se entrecruzam, inclinadas uma a favor e outra contra a inclinação da vertente; ii) as condições mais favoráveis para ocorrência de tombamento aparecem quando a família que mergulha contra a vertente é inclinada a ângulos maiores que (90- $\phi$ ), enquanto a família que mergulha a favor deverá ter inclinações menores que o ângulo de atrito interno; iii) os rumos de mergulho destes planos deverão se situar dentro de $20^{\circ}$ do rumo de mergulho da face da vertente (Fiori \& Carmignani 2009).

MATERIAIS E MÉTODOS O principal equipamento utilizado na pesquisa é o escâner a laser terrestre modelo Cyrax HDS 3000, fabricado pela Leica Geosystems HDS Inc. Este apresenta como principais características sua versatilidade e alta eficiência aliada à alta acurácia, ao incorporar uma capacidade de escanerização de $360^{\circ}$ (horizontal) e $270^{\circ}$ (vertical) por meio de um rápido georreferenciamento. A interface entre usuário e equipamento é o programa Cyclone, desenvolvido pela Cyra, o qual permite a captura de nuvens de pontos, seu processamento e integração com programas convencionais tipo CAD.

A integração e interação de diversas ferramentas computacionais com os equipamentos utilizados na pesquisa permitiu agilizar o processo de aquisição e tratamento de dados na análise da estabilidade dos taludes. Como parte da pesquisa, foi organizado um método para utilização de modelos digitais no estudo da estabilidade de taludes, cujo fluxograma é apresentado na figura 1.

Através do programa Cyclone, foram obtidas as nuvens de pontos georeferenciados, selecionados os pontos de interesse para a pesquisa, geradas as superfícies (meshs), identificadas visual/virtualmente as descontinuidades e obtidas as coordenadas dos pontos que definem as estruturas de interesse.

Uma das vantagens do programa Cyclone é permitir a interação, inclusive através de rotinas próprias, com outros programas tipo CAD, como por exemplo o Autocad, da Autodesk. Em virtude de não ter sido concebido para a análise de taludes e estruturas geológicas, o Cyclone apresenta como limitações os fatos de não permitir a identificação automatizada de planos no modelo de elevação digital (DEM), a definição da orientação espacial de planos definidos pelo usuário ou ainda a não exportação de dados (coordenadas) de pontos de interesse.

\section{Validação do Método}

- Utilização do escâner em um caso real

- Definição de atributos do equipamento (malha, contraste, distância, etc.)

- Aquisição de dados reais e virtuais

- Comparação entre os dados reais e virtuais

- Avaliação dos erros e limitações do método

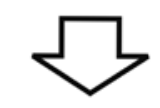

\section{Aplicação do Método a um Estudo de Caso}

- Definição da área de estudo

- Imageamento da área de estudo

- Geração de nuvem de pontos

- Tratamento dos dados

- Identificação de estruturas geológico-geotécnicas

- Obtenção de parâmetros geométricos das estruturas identificadas

- Tratamento dos parâmetros geométricos

- Geração de diagramas estruturais

\section{Análise da Estabilidade de Taludes}

- Tratamento dos dados estruturais

- Análise cinemática do escorregamento de blocos

Figura 1 - Fluxograma do Método de Pesquisa. 
Desta forma, foram necessários os programas Excel, da Microsoft, e Rockworks14, da Rockware para definição das atitudes das estruturas identificadas através de suas coordenadas referenciadas. $\mathrm{Na}$ etapa de processamento dos dados, foram utilizados os programas Stereonett e o Stereo32 do Instituto de Geologia da Universidade de Bochum (Alemanha) para geração dos diagramas estruturais. Empregou-se também o programa Rockworks 14 na geração de diagramas estruturais e histogramas, que permitiram avaliar a consistência dos dados processados, e o Autocad, para o tratamento dos diagramas estruturais no estudo da estabilidade de taludes.

A etapa de campo apresenta como principal resultado uma nuvem de pontos georeferenciados a qual, por hipótese, permite a aquisição de dados estruturais para fins geotécnicos. Todavia, a nuvem de pontos obtida em campo pelo equipamento não está ainda pronta para aplicação, requerendo pré-tratamento dos dados. A primeira etapa deste tratamento consiste na eliminação de pontos imageados que não pertencem ao objeto de interesse (neste caso, a bancada da mina). Assim, são excluídos os pontos que não compõem a figura de interesse.

Esta medida é positiva, pois impede que os pontos que não compõem o objeto anteponham-se ao mesmo, inviabilizando, eventualmente, a visualização de estruturas. Adicionalmente, a nuvem pós-tratamento, em sendo composta por menos pontos, torna-se mais leve no que concerne a seu processamento computacional (rotação de imagens, zoom, escolha de atributos, etc.).

Gerada e tratada a nuvem de pontos, iniciam-se os trabalhos de identificação das estruturas, cujos resultados primários constituem-se nas atitudes das estruturas e correspondente análise, tendo como resultados indiretos os parâmetros necessários para emprego do método (técnica) a esta finalidade. A fim de investigar a acuidade do método, em alguns planos de fratura mais pronunciados, procedeu-se a marcação de planos, através de três pontos referenciados, a curtas (centimétricas) e médias distâncias (métricas).

O reconhecimento de planos, em meio digital, é possibilitado pela rotação da nuvem de pontos, via programa Cyclone. São considerados planos, sucessões de pontos relativamente alinhados sob uma determinada perspectiva, de sorte que sob outra perspectiva estes pontos formem uma malha aproximadamente regular e quadrangular. Na figura 2 pode-se observar um exemplo de aplicação desta metodologia, reconhecendo-se na perspectiva apresentada parte superior da figura os pontos da nuvem que compõem os planos 38 a 41 e, na parte inferior da figura, os padrões de malha regular formado por estes pontos e padrões de malha irregular formados por outras porções da nuvem.

A etapa seguinte do processo constitui-se na marcação das estruturas identificadas, por meio de figuras geométricas conhecidas. Destaca-se aqui o reconhecimento de feições planas as quais foram registradas por meio das coordenadas $(\mathrm{x}, \mathrm{y}, \mathrm{z})$ de três pontos que compõem o plano. A cada plano atribuiu-se um nome de registro, em função da posição de sua bancada de origem e orientação espacial, para fins cadastrais.
Cada um dos planos identificados, representados a partir das coordenadas referenciadas de três de seus pontos, foi submetido a um cálculo para determinação de sua orientação espacial (atitudes dos planos). Desta forma, foi desenvolvida uma planilha no programa Microsoft Excel, para promoção deste cálculo. Paralelamente, os resultados foram lançados no programa Rockworks 14, o qual possui uma rotina para promoção automática deste cálculo. Os resultados foram comparados e os métodos mostraram-se equivalentes.

Os dados estruturais obtidos foram submetidos a uma análise de consistência por meio de histogramas. Esta abordagem permitiu identificar eventuais erros, especialmente erros de transcrição, uma vez que o processo de obtenção das coordenadas (x, y e z) e transcrição para o programa (ou planilha) de cálculo das atitudes se dá de forma manual. Paralelamente, a utilização de histogramas permite avaliar a acuidade (desvio padrão) das medidas obtidas.

De posse das atitudes dos planos, estes foram agrupados em famílias, visando definir padrões estruturais da área. Esta medida permite definir, após a análise geotécnica, quais faces da mina estão mais sujeitas à movimentação (escorregamento) de blocos, quais as formas e volumes destes blocos, permitindo-se tomar medidas preventivas ou mitigadoras à questão.

Área de estudo A área escolhida como objeto do estudo é a mina Saivá, situada no município de Rio Branco do Sul, Paraná, uma jazida de calcário empregado pelo Grupo Votorantim, detentor atual de seus direitos de exploração, na fabricação de cimento.

As rochas aflorantes na mina Saivá, estratigraficamente, pertencem ao Pré-Cambriano paranaense, mais especificamente ao Grupo Açungui, Formação Votuverava. A seqüência litológica descrita por Fiori (1990), denominada Saivá, apresenta três litotipos principais. Inicia-se na base com um filito marrom escuro, com intercalações de níveis milimétricos, esbranquiçados, e eventuais níveis escuros, grafitosos, intercalados. É sucedido por um banco de mármore cinza escuro, relativamente homogêneo e por um pacote de pelitos avermelhados. Entre o mármore e este último, aparece um corpo de metabasitos.

Os trabalhos iniciaram-se pela seleção de uma pequena área da jazida, onde uma bancada de cerca de $200 \mathrm{~m}$ de comprimento por $18 \mathrm{~m}$ de altura foi imageada, o que equivaleu a três horas de aquisição de dados. A escolha da área atentou à dinâmica operacional de exploração da jazida, optando-se por uma zona que não seria explorada em curto prazo, o que possibilitaria que eventuais levantamentos complementares fossem realizados sem prejuízo à geometria do objeto (bancada) selecionado.

O fator limitante desta campanha foi a autonomia das baterias do equipamento, uma vez que não se dispunha de outra fonte de energia no local. Destaca-se que o uso do equipamento para imageamento de áreas externas (minas, por exemplo) requer condições meteorológicas favoráveis (dias ensolarados ou nublados). Gotas de chuva colocam-se como anteparos aos feixes de luz, prejudicando a aquisição dos dados. 

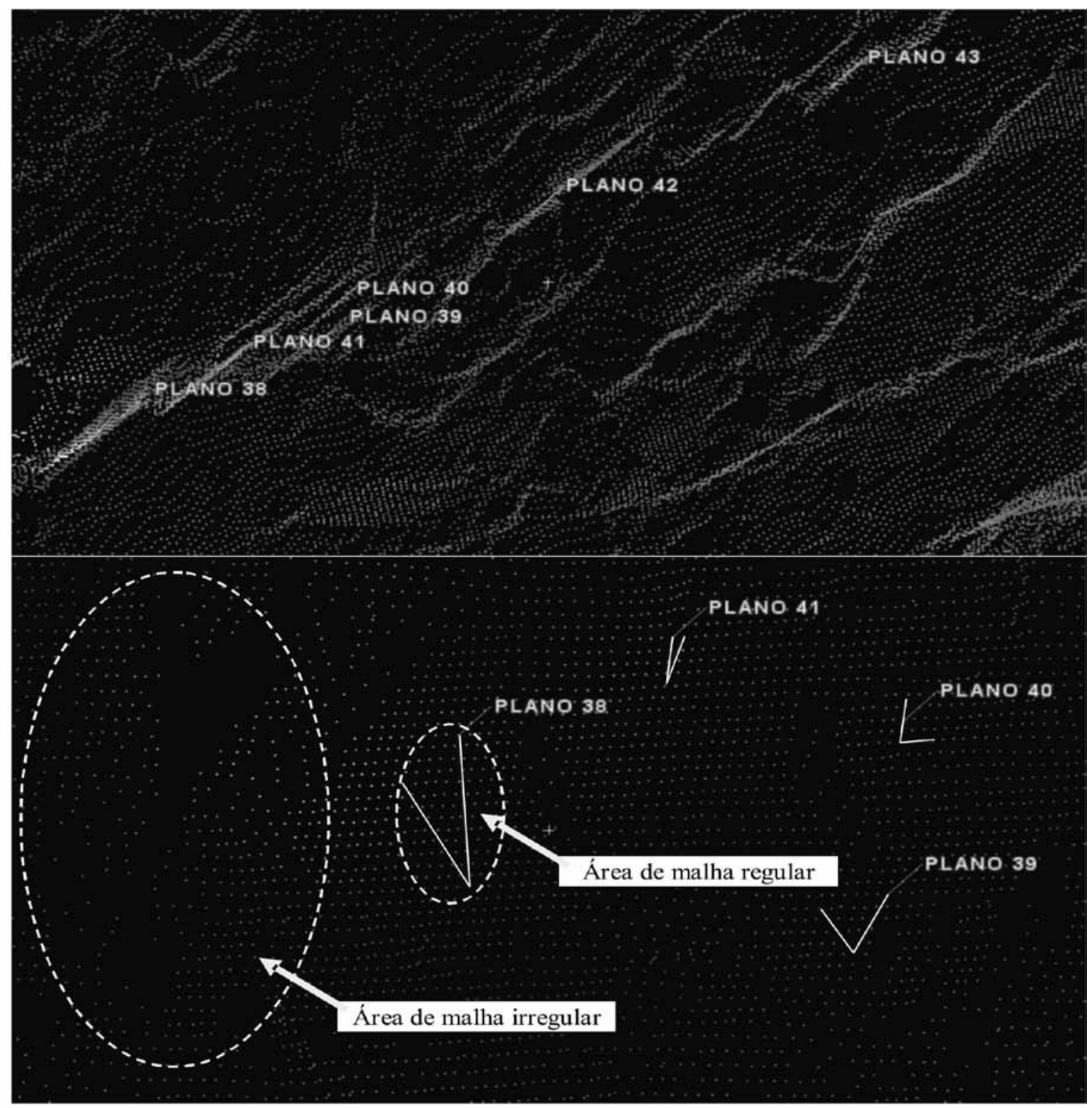

Figura 2 - Detalhe reconhecimento planos 38 a 41 .

ANÁLISE E DISCUSSÃO DOS RESULTADOS Considerações acerca do método proposto Dos experimentos realizados conclui-se que a obtenção adequada dos dados deve, ainda na fase de planejamento, estar atenta a aspectos como: posicionamento e número de estações (locais onde o equipamento será instalado), presença de anteparos e áreas de sombreamento, resolução da malha de pontos e sistema de referência adotado.

A questão do posicionamento das estações deve obedecer às especificações técnicas dos equipamentos, tais como distância do equipamento ao objeto a ser imageado, incidência de luz no objeto, disponibilidade de tempo e autonomia dos equipamentos, resolução da malha, entre outras. O número de estações deve ser suficiente para que não existam falhas significativas na imagem obtida, de forma a não prejudicar futuras análises. Armesto et al. (2009) recomendam a utilização de apenas dois posicionamentos distintos dos equipamentos (estações) como necessárias e suficientes para aplicações da tecnologia em geomorfologia.

Uma vez que o equipamento promove a leitura através da emanação e reflexão de feixes de luz, somente são observados e obtidos dados referentes à superfície do objeto. Assim, a presença de anteparos (árvores, pessoas ou outros) entre o escâner e o objeto impede a obtenção de dados do objeto. Este é considerado, por diversos autores (Sturzenegger \& Stead 2009, Nagalli et al. 2008, Dalmolin \& Dos Santos 2004), como um importante fenômeno a ser observado na realização dos ensaios. De maneira similar, áreas de sombreamento causadas pelo próprio objeto impedem a obtenção de uma malha contínua de pontos, propiciando lacunas nesta. Este efeito pode ser minimizado/solucionado pela utilização de mais de uma estação (posicionamento do equipamento). A incidência de luz no equipamento 
pode ser ajustada internamente no programa (Cyclone, por exemplo) parametrizando-se o contraste.

A resolução da malha a ser obtida é função das características de cada escâner. Em geral, os equipamentos mais utilizados no mercado para uso externo permitem precisões da ordem de milímetros, em função da distância escâner-objeto. Todavia, quanto maior a resolução da malha, maior o tempo de aquisição destes dados. Assim, escanerizações a céu aberto, por questões práticas, geralmente são promovidas com resoluções da ordem de centímetros (2 a 5cm). Pesci et al. (2007), objetivando a obtenção de um modelo digital topográfico, efetuaram a comparação entre resultados obtidos a partir de escâneres a laser terrestre com equipamentos de fotogrametria aérea e concluíram que as duas ferramentas apresentam-se como complementares e possibilitam a obtenção de um modelo bastante acurado, em diferentes escalas (o escâner, em escala de detalhe).

A versatilidade do método está condicionada à interação entre o programa de processamento e tratamento da nuvem de pontos gerada e os demais programas. Especialmente quando se trata de análises estruturais, programas específicos são necessários e, caso não exista esta compatibilidade entre os programas, $o$ emprego das imagens digitais torna-se limitado, como relatado por Schueremans \& Genechten (2009).

Uma das vantagens que se apresenta no pósprocessamento dos dados, apontada por Sturzenegger \& Stead (2009) e Nagalli et al. (2008), é a possibilidade de se analisar estruturas geológicas sob diversas perspectivas. Os programas utilizados, em geral, permitem uma visualização completa da malha gerada, permitindo a rotação e zoom da nuvem de pontos. A visualização de detalhe pode possibilitar a identificação de estruturas ou processos até então desconhecidos.

Sturzenegger \& Stead (2009) concluíram, através da comparação de atitudes de descontinuidades obtidas a partir de escâner terrestre e clinômetros (compass clinometer), que os erros máximos associados a medidas de mergulho e direção de mergulho são de $4^{\circ}$ e $8^{\circ}$, respectivamente. Desta forma, a acurácia dos dados obtidos com o escâner a laser permite que estes sejam utilizados para fins geotécnicos, considerando a natural variabilidade da orientação destas descontinuidades. Os maiores erros de medição estiveram associados às superfícies curvas, situações em que é inevitável, em termos práticos, esta diferença (Sturzenegger \& Stead 2009).

Definida a área a ser imageada, o escaneamento é realizado por etapas, separando-se a aquisição de dados por quadros no programa Cyclone. Uma consideração importante, relatada Pierin et al. (inédito) e Sturzenegger \& Stead (2009), que facilita o georeferenciamento dos dados e minimiza efeitos de distorção da imagem, refere-se ao posicionamento e direcionamento do equipamento em relação ao objeto a ser imageado e ao horizonte de imageamento. No que toca ao posicionamento do equipamento, este não deve situar-se a uma distância superior a $100 \mathrm{~m}$ da bancada (objeto) a ser imageada (limitação de alcance do aparelho). Nesta etapa do estudo, o equipamento posicionou-se a uma distância da ordem de $80 \mathrm{~m}$ da bancada.

No que concerne ao direcionamento do equipamento, este deve estar orientado/apontado para o norte verdadeiro, através de bússola. Esta situação evita a necessidade de rotação de coordenadas para novo sistema de eixos através de processamento posterior e erros associados. O equipamento, acoplado a um tripé, é fixado junto ao solo e calado por meio de bolha niveladora. Cabos de energia são ligados às baterias e cabos de rede conectam o equipamento ao computador que processa o armazenamento dos dados obtidos. O procedimento, em detalhe, é descrito em Mezzomo (2007).

No consentâneo ao horizonte de imageamento, considerando que os equipamentos/programas permitem justaposição de imagens, deve-se promover o imageamento por etapas (fatias). Este se justifica pelo fato de que a distância entre o objeto (bancada da mina) e o aparelho (escâner) é variável, por não se tratar de uma mina de formato circular. Ao contrário, a imagem adquirida poderia apresentar distorções significativas decorrentes deste distanciamento diferenciado. Esta medida, ao segmentar o tempo total de escanerização, minimiza os riscos de aborto da operação de aquisição de dados e conseqüente perda de informações. Assim, a área imageada foi composta por "fatias" de 30 a 40m de largura. Situação semelhante se aplicaria à direção vertical, embora, nesta etapa da pesquisa, tenha-se definido a altura da imagem como aproximadamente igual à altura da bancada imageada $(18 \mathrm{~m})$.

Sempre que possível, é desejável o emprego do imageamento de uma mesma parede sob dois posicionamentos diferentes do laser escâner. Esta medida visa cobrir eventuais zonas de sombreamento, gerando uma superfície tridimensional mais próxima da realidade. Para tal, vale-se de marcos de referência, dispostos adequadamente junto à parede imageada com vistas a compatibilizar posteriormente ambas as imagens. A interface de aquisição e edição dos dados se dá pelo programa Cyclone, responsável pela operação do aparelho. Nesta etapa inicial, devido às limitações de autonomia de energia do equipamento não foi possível o emprego desta técnica.

No que tange à resolução da imagem, ainda antes do início dos levantamentos, é necessário parametrizar junto ao escâner a laser a malha de aquisição dos dados (distância entre pontos). Esta foi definida, em função de limitações de tempo de aquisição de informação e de bateria do aparelho, como uma malha que variou de $1,7 \times 1,7 \mathrm{~cm}$ a $2,0 \times 2,0 \mathrm{~cm}$. Quanto maior a malha, menor o tempo de aquisição de dados e pior a potencial qualidade da imagem adquirida. Para fins de uma avaliação preliminar, julgou-se proveitoso, em função do tamanho da mina e limitações do método, uma resolução de imagem que abrangesse grande área (para representatividade de estruturas), sem perda significativa, por hipótese, de qualidade de dados.

Outro parâmetro de entrada no equipamento é o contraste, definido para a situação outdoor como igual a 8. Este parâmetro é função das condições de iluminação (insolação) no local, cores associadas à imagem, 
etc., tendo sido definido empiricamente no local. A resolução da câmera digital foi definida em 1 megapixel (máxima do equipamento). Nesta campanha preliminar imageou-se parte da face leste da mina (bancada inferior). A escolha do local imageado considerou o plano de lavra, optando-se por uma face desativada, por uma questão de segurança e reprodutibilidade dos ensaios.

Paralelamente, foram obtidas em campo algumas atitudes de estruturas representativas, as quais serviriam posteriormente como base de comparação entre estruturas reais e digitais e validação do método.

Resultados da aplicação ao estudo de caso $O$ primeiro resultado consiste na nuvem de pontos obtida pelo imageamento de uma bancada através do escâner a laser. A nuvem relativa a este trabalho é composta por 10.352 .528 pontos referenciados, distribuídos em 21 faixas (scans).

Inerente à tecnologia empregada, cada um destes pontos (pixels) que compõem a nuvem tem a si associados, além de suas coordenadas ( $\mathrm{x}, \mathrm{y}$ e $\mathrm{z}$ ou $\mathrm{N}$, $\mathrm{E}, \mathrm{El})$ no sistema, características como intensidade de reflexão do laser e cor, pela superposição da fotografia à imagem, possibilitando a geração de diferentes imagens, tais como as apresentadas na figura 3 .

Com base nesta nuvem de pontos referenciada, foi dado início à etapa seguinte do trabalho, que consistiu na triagem dos dados obtidos, a fim de eliminar os pontos da imagem que não compunham propriamente a bancada objeto de análise. A atividade de "limpeza" da imagem permitiu agilizar os trabalhos computacionais (processamentos) com a nuvem de pontos, pela diminuição da quantidade de dados, restringindo-os a dados úteis.

Foram identificados seis grupos de planos, posteriormente agrupados em famílias. Os detalhes desta forma de investigação são apresentados na figura 4 . Cumpre salientar que as marcações das estruturas se deram em duas frentes de lavra diferentes, face leste e face norte da jazida, de forma a aproveitar ao máximo os dados obtidos na campanha preliminar para identificação das estruturas mais marcantes no maciço. $\mathrm{O}$ fato de estas duas faces serem ortogonais facilita a visualização de famílias de descontinuidades não facilmente identificadas nesta ou naquela face.

Os resultados das medições digitais revelaram uma boa precisão nas medidas obtidas, facilitados pela boa resolução da imagem, de modo que os desvios padrão calculados revelam-se similares aos obtidos por meio dos métodos convencionais em geologia. Desta forma, confirmam-se os resultados obtidos por Sturzenegger \& Stead (2009), como apresentado na revisão bibliográfica, ratificando-se que resoluções da malha de escanerização da ordem de $2 \times 2 \mathrm{~cm}$ são suficientes para a análise.

Os dados estruturais obtidos foram submetidos a uma análise de consistência por meio de histogramas. Esta abordagem permitiu identificar eventuais erros, especialmente erros de transcrição, uma vez que o processo de obtenção das coordenadas (x, y e z) e transcrição para

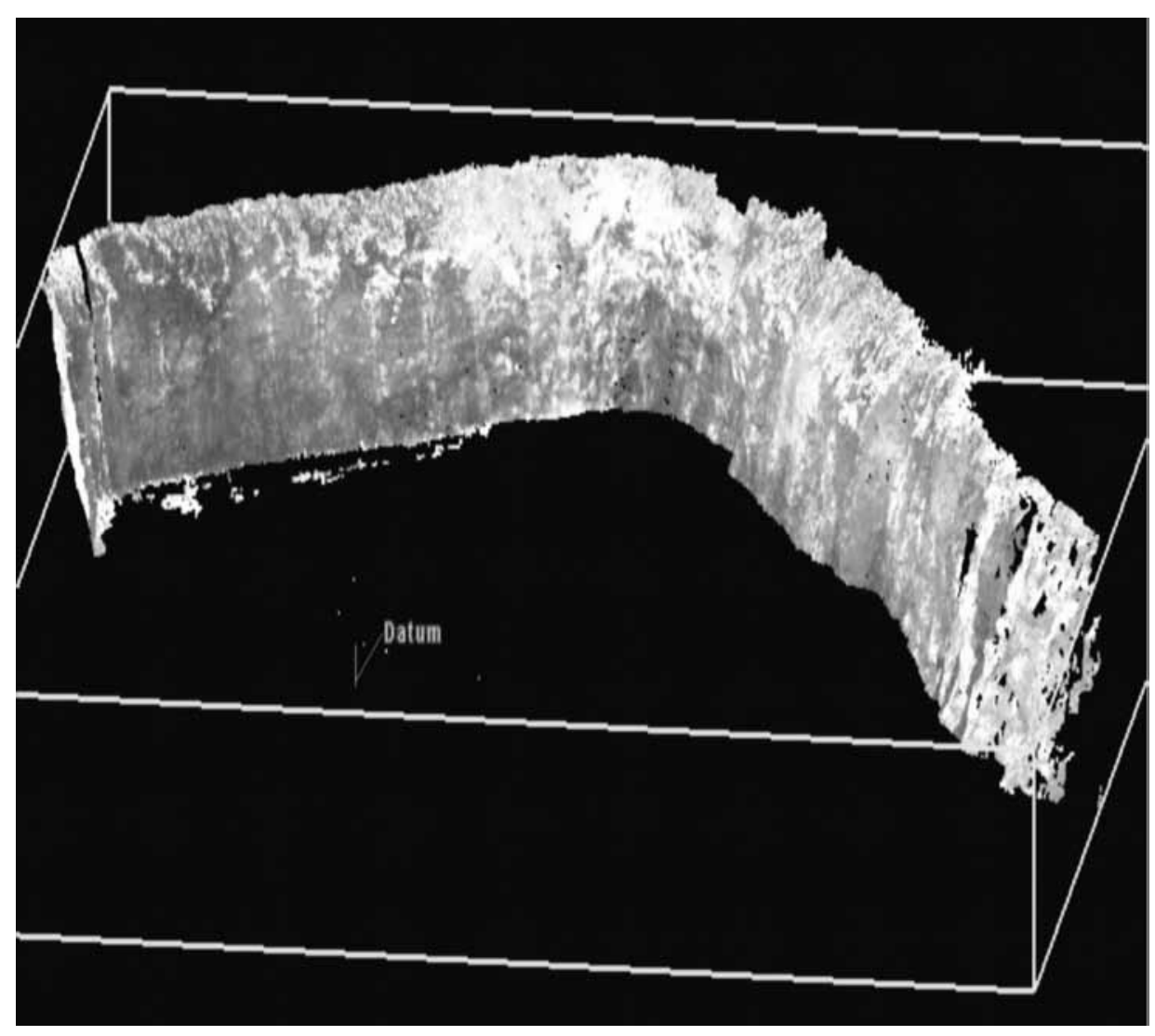

Figura 3 - Perspectiva da nuvem de pontos, com sobreposição de fotografia. 


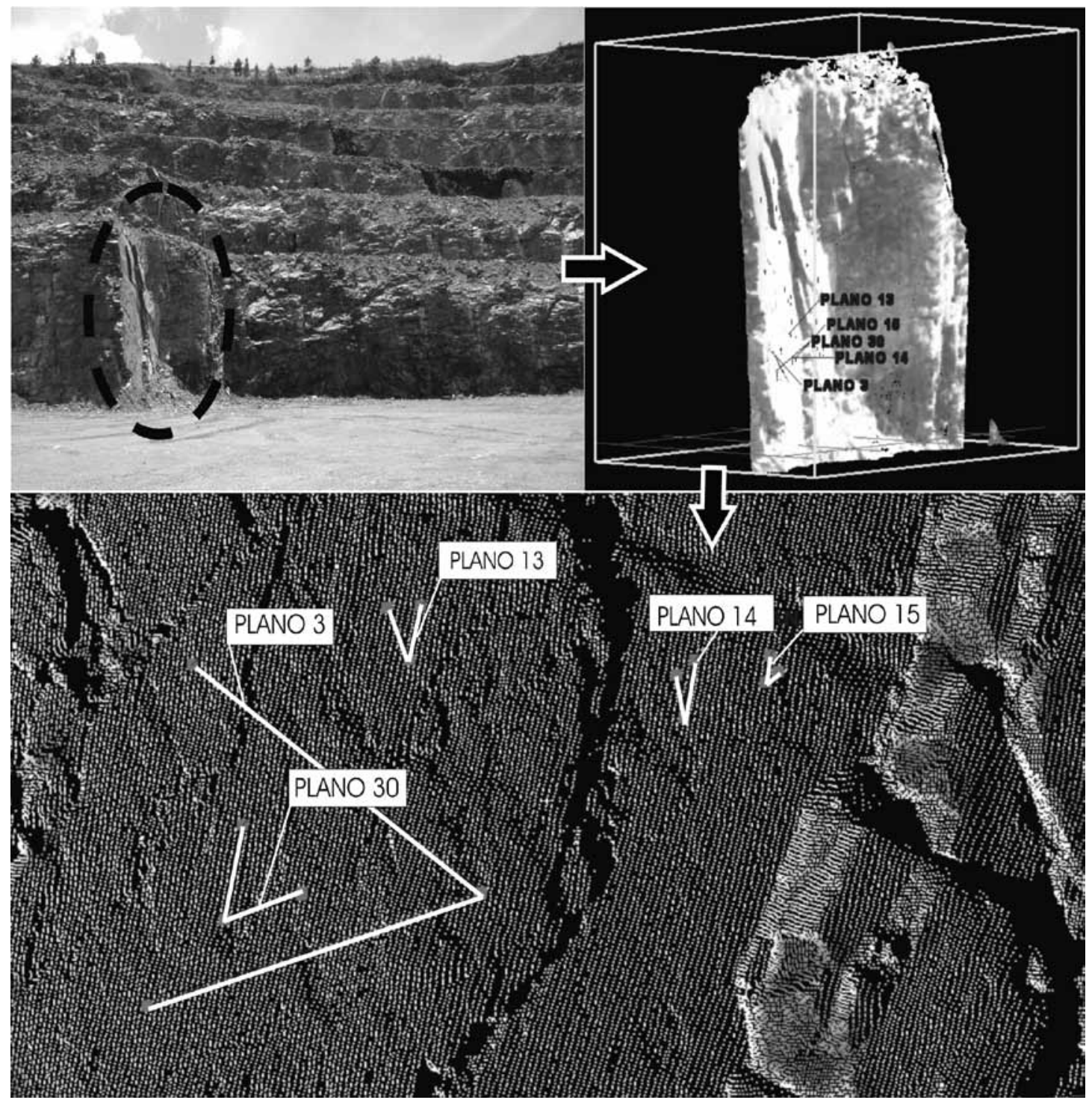

Figura 4 - Plano de fratura com investigação de detalhe.

o programa de cálculo das atitudes se dá de forma manual. Paralelamente, a utilização de histogramas permite avaliar a acuidade (desvio padrão) das medidas obtidas.

Outro aspecto a se considerar é a rugosidade dos planos, quanto mais rugoso o plano, piores são os resultados. Esta questão pode ser minimizada empregando-se o maior distanciamento entre os pontos de identificação do plano. Superfícies de ruptura curvas também dificultam a aplicação da técnica.

No que concerne ao distanciamento entre os pontos que definem os planos de ruptura, pôde-se concluir que quanto maior o espaçamento entre os pontos, menos variável e mais precisa são as medidas. Em geral, quanto mais distantes os pontos, mais representativas se revelam as medidas. Assim, é recomendada a tomada de pontos o mais distantes possível para definição da atitude dos planos de ruptura, embora nem sempre faces extensas estejam presentes. Contudo, esta alternativa apresenta limitações intrínsecas ao método, pois somente se pode escolher pontos que pertençam à face exposta imageada pelo escâner. Assim, a maior distância permitida entre os pontos será, geometricamente, a maior diagonal do plano de ruptura em questão. No caso de superfícies de ruptura irregulares ou curvilíneas, grandes variações nas atitudes são esperadas, inerentes à natureza destas estruturas.

Padrão estrutural da área imageada Identificadas e medidas as estruturas no modelo digital, pode-se inferir um padrão estrutural da área imageada. Os grupos de planos foram lançados, pela utilização do programa Rockworks 14, nos diagrama de Schmidt-Lambert e de rosetas, visando uma melhor visualização dos padrões estruturais. Nota-se na figura 5 que as estruturas identificadas em meio digital apresentam uma orientação predominantemente NE, com mergulhos que variam de médios a altos ângulos, tanto para SE, quanto para NW. Estes resultados mostram-se compatíveis com os registrados por Figueira (1999) e pelos levantamentos atuais dos técnicos da mina. 


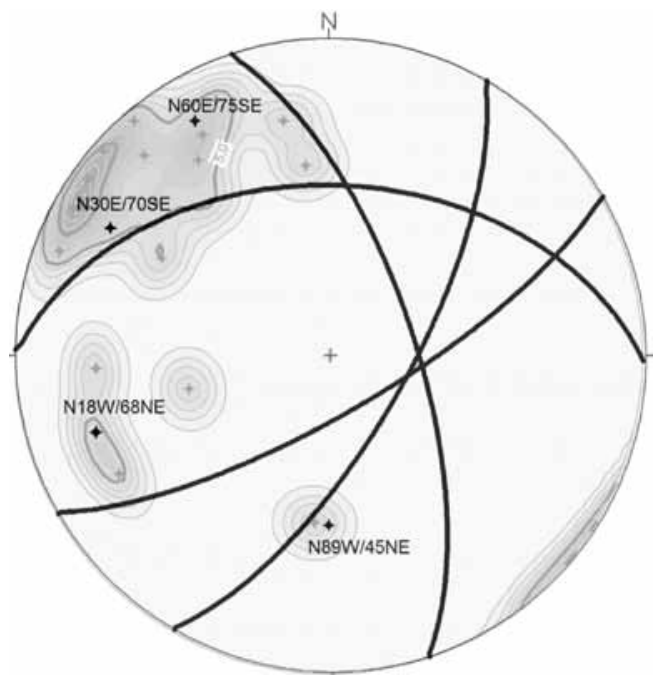

Figura 5 - Projeção ciclográfica e polar da concentração máxima de pólos e suas respectivas estruturas planas médias identificadas nos afloramentos, obtidas no imageamento.

Análise cinemática dos taludes em rocha A análise cinemática dos taludes em rocha da mina Saivá iniciase com a representação, por meio de projeção ciclográfica, das famílias de planos mais penetrativas identificadas nos levantamentos geológico-estruturais digitais.

A exemplo da determinação das atitudes das estruturas geológicas, a medição da inclinação das vertentes das bancadas da mina de Saivá aconteceram por meio das coordenadas referenciadas de três pontos. Fo- ram tomadas três medidas e adotado um valor médio. Os valores obtidos para estas inclinações variaram de 64,1 a $75,1^{\circ}$. Assim, para efeitos de cálculo, adotou-se uma inclinação média de $70^{\circ}$ para as vertentes.

Para efeitos de cálculo, já que não se dispõe de informações bibliográficas específicas sobre as características geomecânicas da rocha, foi adotado um ângulo de atrito interno igual a $30^{\circ}$.

Considerando-se os dados supracitados, elaborou-se um overlay para combinação com os estereogramas de dados, a fim de avaliar a estabilidade dos taludes quanto a movimentações segundo estruturas planares (Fig. 6).

Os diagramas gerados revelam que a única família de estruturas que se insere na zona de instabilidade é a família N89W/45NE e, desta forma, seria o conjunto de estruturas com as quais se deveria ter alguma preocupação em termos geotécnicos, no que toca a seu escorregamento segundo estruturas planares. Desta forma, em havendo movimentação de blocos esta aconteceria possivelmente segundo escorregamento planar.

Empregando-se o teste de Markland (1972), procedeu-se a análise de deslizamentos em cunha e tombamento, considerando-se que a exploração da mina desenvolveu escavações cuja direção das vertentes são N40E/70NW, N50W/70NE, N50W/70SE e $\mathrm{N} 30 \mathrm{E} / 70 \mathrm{SE}$ e inclinações médias de $70^{\circ}$. Os resultados são apresentados na figura 7 .

Depreende-se dos diagramas executados que as vertentes das escavações com orientação N40E/70NW, consoante às estruturas planares levantadas e lançadas nos estereogramas, mostram-se susceptíveis a deslizamentos em cunha originados a partir de associações de planos de fratura das famílias N18W/68NE e N89W/45NE, sendo relevante uma análise mais deta-

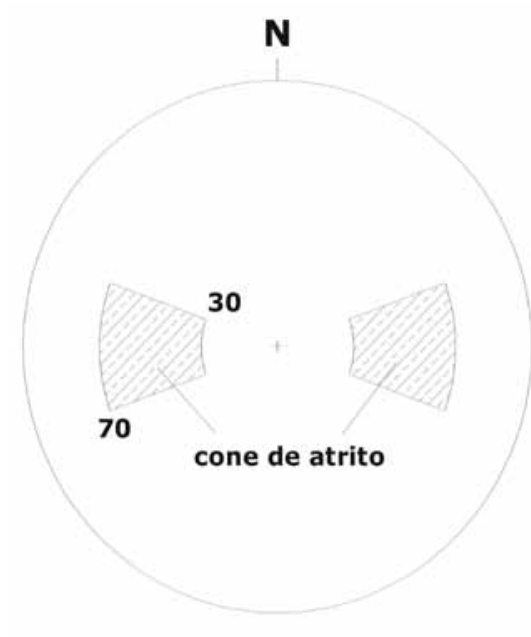

(A)

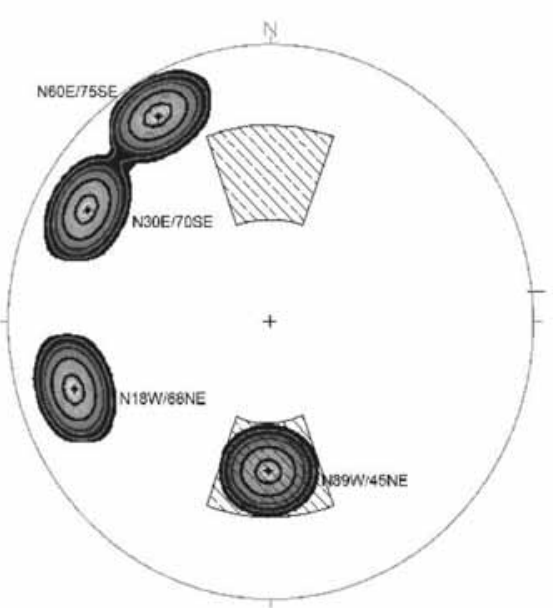

(B)

Figura 6 - (A) overlay contendo o cone de atrito, preparado para uma vertente de $70^{\circ}$ de inclinação e $30^{\circ}$ de ângulo de atrito interno e (B) sua aplicação, para as estruturas imageadas. 
lhada. Outra constatação é que nenhuma das associações entre as descontinuidades identificadas através do modelo digital dá origem a blocos com possibilidade de deslizamento em cunha, para escavações na direção N30E/70SE. Assim, estas vertentes mostram-se seguras do ponde de vista de deslizamentos em cunha.

No que toca às vertentes de orientação N50W/70NE, conclui-se que estas apresentam a possibilidade de deslizamentos em cunha para associações das famílias de juntas N30E/70SE e N89W/45NE. Assim, também é recomendável uma análise mais detalhada destes blocos. Constata-se também que nenhuma das associações entre as descontinuidades identificadas através do modelo digital dá origem a blocos com possibilidade de deslizamento em cunha, para escavações na direção N50W/70SE.

Em relação à possibilidade de tombamento de blocos, as orientações dos taludes das bancadas e a disposição espacial das famílias de estruturas identificadas, quando analisadas de forma conjugada, não permitem um arranjo que favoreça a ocorrência deste processo. A análise dos diagramas revela que os altos ângulos de mergulho das famílias de estruturas identificadas não atendem às condições fundamentais para o tombamento de blocos, apresentadas em Fiori \& Carmignani (2009).
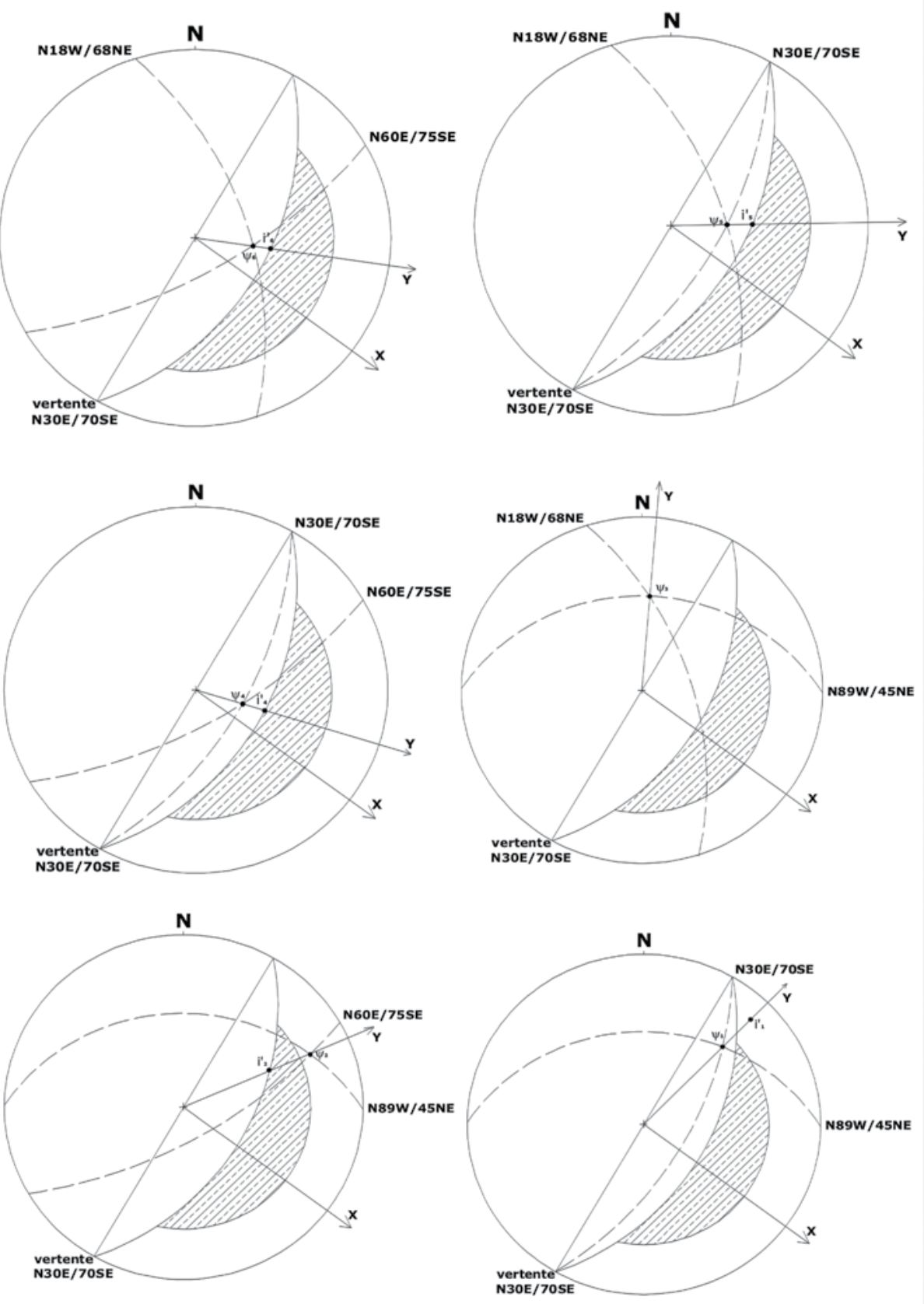

Figura 7 - Análise de deslizamento em cunha para as escavações N30E/70SE. 
CONCLUSÕES E RECOMENDAÇÕES O estudo geotécnico de taludes em rocha possibilita avaliar sua estabilidade, garantindo sua segurança para lindeiros, e, no âmbito industrial, permite a elaboração de planos de lavra otimizados, no que toca ao aproveitamento dos minérios, economia e segurança dos trabalhos. $\mathrm{O}$ emprego do escâner a laser revelou amplo potencial de aplicação no estudo da estabilidade de taludes.

Destacam-se como principais vantagens da utilização deste novo método a possibilidade de se realizar levantamentos geológico-estruturais à distância, especialmente em áreas onde o acesso é dificultoso ou perigoso, a possibilidade de se trabalhar e consultar a posteriori os dados estruturais, dirimindo eventuais dúvidas e complementando a alimentação de modelos matemático-computacionais, a boa precisão na tomada de atitudes de planos de fratura, entre outros.

Outro ponto positivo importante está associado à grande quantidade de dados estruturais que podem ser adquiridos e processados simultaneamente, aumentando o grau de confiabilidade das interpretações.

Como limitações na aplicação dessa tecnologia, destaca-se que os levantamentos para aquisição dos dados devem acontecer em condições meteorológicas favoráveis, isto é, sem chuva, e que o equipamento (Cyrax HDS-3000) não deve estar posicionado a distâncias superiores a $100 \mathrm{~m}$ do objeto a ser imageado. Depreendese que não podem existir anteparos (árvores, pessoas, outros objetos) dispostos entre o escâner e o objeto, sob pena de a imagem adquirida apresentar falhas no regis- tro de dados. Por este motivo é recomendada a utilização de pelo menos duas estações de escanerização, não necessariamente simultâneas, para análise.

Os resultados obtidos revelam que a ferramenta mostra-se bastante vantajosa e precisa na medição de atitudes de planos de ruptura. A amplitude de variação das medidas de atitudes tomadas digitalmente mostrou-se compatível com as observadas em campo e a possibilidade de se trabalhar simultaneamente grande quantidade de dados mostra-se um diferencial. As direções preferenciais das medidas obtidas em campo são compatíveis com os resultados da análise digital.

De posse do modelo digital da mina, foram levantados dados geológico-estruturais que subsidiaram a análise de escorregamentos de taludes, segundo movimentos planares ou deslizamento em cunha, em função da disposição espacial das famílias de juntas reconhecidas na nuvem de pontos gerada pelo escâner. Assim, os resultados do método permitiram a análise cinemática da vertente imageada.

O objetivo inicial de validar a aplicação desta nova tecnologia foi alcançado, respondendo-se às hipóteses iniciais ao depreender-se que resoluções de malha de levantamento da ordem de centímetros ( 2 a $5 \mathrm{~cm}$ ) são suficientes para aplicações em estudos geotécnicos de taludes em rocha. Assim, esta nova ferramenta apresenta imediata possibilidade de aplicação em otimização de planos de lavra, escavações de túneis, cortes de rodovias, monitoramento de encostas, entre outras atividades em geotecnia.

\section{Referências}

Abellán A., Vilaplan J.M., Martínez J. 2006. Application of a long-range Terrestrial Laser Scanner to a detailed rockfall study at Vall de Núria (Eastern Pyrenees, Spain). Engineering Geology, 88:136-148.

Armesto J., Ordóñez C., Alejano L., Arias P. 2009. Terrestrial laser scanning used to determine the geometry of a granite stability analysis purposes. Geomorphology, 106:271-277.

Barber D., Mills J., Voysey-Smith S. 2008. Geometric validation of a ground-based mobile laser scanning system. ISPRS Journal of Photogrammetry \& Remote Sensing, 63:128-141.

Barnea S. \& Filin S. 2008. Keypoint based autonomous registration of terrestrial laser point-clouds. ISPRS Journal of Photogrammetry \& Remote Sensing, 63:19-35.

Beraldin J.A., Blais F., Boulanger P., Cournoyer L., Domey J., El-Hakim S.F., Godin G., Rioux M., Taylor J. 2000. Real World modelling through high resolution digital 3D imaging of objects and structures. ISPRS Journal of Photogrametry and Remote Sensing, 55:230-250.

Cote J., Widlowski J., Fournier R.A., Verstraete M.M. 2009. The structural and radiative consistency of three-dimensional tree reconstructions from terrestrial lidar. Remote Sensing of the Environment, 113:1067-1081.

Dalmolin Q. \& Dos Santos D.R. 2004. Sistema lasercanning: conceitos e princípios de funcionamento. 3ed. Curitiba,
Departamento de Geomática, Ed. UFPR, 97 p.

Dunning S.A., Massey C.I., Rosser N.J. 2009. Structural and geomorphological features of landslides in the Bhutan Himalaya derived from Terrestrial Laser Scanning. Geomorphology, 103:17-29.

Entwistle J.A., Mccaffrey K.J.W., Abrahams P.W. 2009. Three-dimensional (3D) visualization: the application of terrestrial laser scanning in the investigation of historical Scottish farming townships. Journal of Archaeological Science, 36:860-866.

Fardin N., Stephansson O., Jing L. 2001. The scale dependence of rock joint surface roughness. International Journal of Rock Mechanics \& Mining Sciences, 38:659-669.

Fardin N., Feng Q., Stephansson O. 2004. Application of a new in situ 3D laser scanner to study the scale effect on the rock joint surface roughness. International Journal of Rock Mechanics \& Mining Sciences, 41:329-335.

Figueira E.G. 1999. Modelagem geoestatística da mina Saivá, Rio Branco do Sul, PR. Dissertação de Mestrado, Universidade Federal do Paraná, 125p.

Fiori A.P. 1990. Tectônica e estratigrafia do Grupo Açungui a norte de Curiiba. Tese de livre-docência, Instituto de Geociências, Universidade de São Paulo, 261 p.

Fiori A.P. \& Carmignani L. 2009. Fundamentos de mecânica dos solos e das rochas: aplicações na estabilidade de taludes. $2^{\mathrm{a}}$ ed., Curitiba, Editora UFPR, 604 p. 
Franceschi M., Teza G., Preto N., Pesci A., Galgaro A., Girardi S. 2009. Discrimination between marls and limestones using intensity data from terrestrial laser scanner. ISPRS Journal of Photogrammetry and Remote Sensing, 64:1-7.

Lato M., Diederichs M.S., Hutchinson D.J., Harrap R. 2009. Optimization of LIDAR scanning and processing for automated structural evaluation of discontinuities in rockmasses. International Journal of Rock Mechanics \& Mining Sciences, 46:194-199.

Lerma J.L., Navarro S., Cabrelles M., Villaverde V. 2009. Terrestrial laser scanning and close range photogrammetry for 3D archaeological documentation: the Upper Palaeolithic Cave of Parpalló as a case study. Journal of Archaeological Science, 37:1-9.

Li J., Guo Y., Zhu J., Lin X., Xin Y., Duan K., Tang Q. 2007. Large depth-of-view portable three-dimensional laser scanner and its segmental calibration for robot vision. Optics and Lasers in Engineering, 45:1077-1087.

Markland J.T. 1972. A useful technique for estimating the stability of rock slopes when the ridge wedge sliding type of failure is expected. Imperial College Rock Mechanics Research Report, 19:1-10.

Mezzomo E. 2007. Integração de modelos numéricos para caracterização do arcabouço geológico $3 D$ na porção Centro-Leste da Bacia do Paraná. Dissertação de Mestrado, Universidade Federal do Paraná, 98 p.

Meunier P., Tack D., Ricci A., Bossi L., Angel H. 2000. Helmet accommodation analysis using 3D laser scanning. Applied Ergonomics, 31:361-369.

Nagalli A., Fiori A.P., Rostirolla S.P., Pierin A.R.H. 2008. Utilização de 3D laser scanner para aquisição de dados geológico-estruturais no desenvolvimento de modelo geomecânico
- Estudo de caso da Mina Saivá, Paraná. In: Congresso Brasileiro de Geologia, 44, Curitiba, Anais, p. 675.

Paronuzzi P. \& Serafini W. 2009. Stress state analysis of a collapsed overhanging rock slab: A case study. Engineering Geology, 108:65-75.

Pesci A., Massimo F., Conforti D., Loddo F. 2007. Integration of ground-based laser scanner and aerial digital photogrammetry for topographic modeling of Vesuvio volcano. Journal of Volcanology and Geothermal Research, 162:123-138.

Schueremans L. \& Genechten B.V. 2009. The use of 3Dlaser scanning in assessing the safety of masonry vaults - A case study on the church of Saint Jacobs. Optics and Lasers in Engineering, 47:329-335.

Staiger R. 2003. Terrestrial laser scanning - technology, systems and applications. In: FIG Regional Conference, Marrakech, Morocco, 2-5 December, Proc. Disponível em: http://www.fig.net/pub/morocco/proceedings/TS12/ TS12_3_staiger.pdf. Acesso em 15 de setembro de 2009.

Sturzenegger M. \& Stead D. 2009. Close-range terrestrial digital photogrammetry and terrestrial laser scanning for discontinuity characterization on rock cuts. Engineering Geology, 106:163-182.

Turanboy A., Gökay M.K., Ülker E. 2008. An approach to geometrical modeling of slope curves and discontinuities. Simulation Modelling Practice and Theory 16:445-461.

Yoon J., Sagong M., Lee J.S., Lee K. 2009. Feature extraction of a concrete tunnel liner from 3D laser scanning data. NDT\&E International, 42:97-105.

Manuscrito ID 16442

Submetido em 18 de dezembro de 2009 Aceito em 05 de novembro de 2010 